\section{A $\mathbf{R}_{\text {Eview }}$}

\section{Chandra Shekhar}

Department of Veterinary Public Health and Epidemiology, College of Veterinary Science and Animal Husbandry, N.D. University of Agriculture and Technology, Kumarganj, Faizabad (U.P.) India Email : cshekharvph@gmail.com

\section{Impact of brucellosis on health and economy}

Chandra Shekhar

\begin{abstract}
Brucellosis is one of the world's major zoonoses that still are of veterinarian, public health and economic concern in many parts of the world. It is an important zoonosis in humans in many parts of the world especially in the Mediterranean countries of Europe, north and east Africa, the Middle East, south and central Asia and Central and South America and yet it is often unrecognized and frequently goes unreported. Over half a million new cases occurs annually and prevalence rates in some countries exceeding ten cases per 100000 population. Brucellosis has been constantly ranked among the most economically important zoonoses worldwide with multiple economic impacts attributable to human, livestock and wildlife disease. Human acquires Brucella infection directly or indirectly from animal sources. Brucellosis is a sub-acute or chronic disease which may affect many species of animals and cause placentitis followed by abortion in the pregnant female, usually during the last third of pregnancy, and epididymitis and orchitis in the male. The disease usually manifests itself as an acute febrile illness which may persist and progress to a chronic disease with severe complications. Animal brucellosis can be prevented, controlled and eradicated by farm hygiene and sanitation, restricted movement, prevention of contact, test and isolation/slaughter, mass vaccination, treatment and surveillance. Human brucellosis can be prevented and controlled by controlling and eliminating the diseases in animal reservoir and health education of the public working at high risk areas.
\end{abstract}

Key words : Brucellosis, Economic, Epidemiology, Health, Management

How to cite this paper : Shekhar, Chandra (2018). Impact of brucellosis on health and economy. Vet. Sci. Res. J., 9(1\&2) : 37-44, DOI : 10.15740/HAS/VSRJ/9.1and2/37-44.Copyright@ 2018: Hind AgriHorticultural Society.

Paper History : Received : 23.12.2017; Accepted : 30.09.2018 